\title{
Food Frequency Index as a Measure of Diet Quality in Non-Frail Older Adults
}

\author{
Heinz Freisling Ibrahim Elmadfa \\ Department of Nutritional Sciences, University of Vienna, Vienna, Austria
}

\section{Key Words}

Diet quality · Non-frail older adults · Nutritional risk

factors $\cdot$ Food Frequency Index

\begin{abstract}
Aims: The objective of the present work was to identify factors associated with diet quality in non-frail older adults measured with the Food Frequency Index (FFI). Methods: In a cross-sectional survey, data from a qualitative 28 -item food-frequency questionnaire was used to calculate the FFI in a sample of 444 adults aged 55 years and older living in Vienna, Austria. Results: A higher FFl, indicating a favorable diet quality, was obtained for women as compared to men, younger and better-educated subjects ( $p$ for all $<0.01$ ). Dietary supplement users $(p=0.04)$ and subjects being physically active $(p<0.01)$ showed significantly higher FFI scores. Eating difficulties $(p=0.04)$ and gastrointestinal problems $(p=0.03$ ) were found to be risk factors for poor diet quality as measured with the FFI, whereas the number of prescribed daily medications was not associated with diet quality ( $p$ for trend $=0.77$ ). Conclusions: Differences in diet quality are evident in subgroups of non-frail older adults living in Vienna as defined by societal factors, such as education, lifestyle-related factors, such as being physically active and health-related factors, such as oral health.
\end{abstract}

Copyright $\odot 2008$ S. Karger AG, Basel

\section{Introduction}

Indexes of overall diet quality are described in the literature as an alternative approach of using single nutrients to examine diet-disease relationships or to assess the diet quality of a population and population subgroups [1-3].

Information about dietary habits of populations is needed to identify nutrition problems or factors associated with poor diet quality so that effective intervention measures can be established. However, representative data on dietary habits of a population is difficult to collect and depending on the level of detail it can be an elaborative and expensive task. It is even more difficult to measure diet stratified by sociodemographic and other characteristics of population subgroups.

A recently developed index of diet quality, the Food Frequency Index (FFI), has shown the ability to discern subgroups of a population into low- or high-risk dietary behaviors with reasonable validity [unpubl. data].

The objective of the present work was to identify potential factors associated with diet quality in non-frail older adults measured with the FFI.

\section{KARGER}

Fax +4161306 1234 E-Mail karger@karger.ch www.karger.com
Dr. Heinz Freisling

University of Vienna, Department of Nutritional Sciences

Althanstrasse 14

AT-1090 Wien (Austria)

Tel. +43 14277549 12, Fax +43 1427795 49, E-Mail heinz.freisling@univie.ac.at 
Table 1. Mean FFI scores by sociodemographic characteristics of the subjects $(n=306)$

\begin{tabular}{lrlc}
\hline Characteristics & $\mathrm{n}^{1}$ & $\mathrm{FFI}^{2}$ & $\mathrm{p}$ value \\
\hline $\begin{array}{l}\text { Gender } \\
\quad \text { Female }\end{array}$ & 259 & $35.1(0.9)$ & \\
$\quad$ Male & 41 & $31.7(0.3)$ & $<0.001$ \\
Age & & & \\
$\quad$ 55-65 years & 98 & $35.3(0.5)$ & \\
$\quad$ 66-75 years & 73 & $35.9(0.6)$ & 0.44 \\
$\quad$ 76-85 years & 86 & $34.8(0.6)$ & 0.58 \\
$\quad$ >66 years & 43 & $31.3(0.8)$ & $<0.001$ \\
Education & & & \\
$\quad$ Low & 166 & $33.6(0.4)$ & \\
$\quad$ Middle & 64 & $35.6(0.7)$ & 0.01 \\
$\quad$ High & 29 & $36.1(1.0)$ & 0.02 \\
$\quad$ Other & 40 & $36.1(0.9)$ & 0.01 \\
Dwelling & & & \\
$\quad$ Private home & 202 & $35.1(0.5)$ & \\
$\quad$ Nursing home & 98 & $33.7(0.6)$ & 0.12 \\
\hline
\end{tabular}

${ }^{1}$ Values are not always equal to total number of subjects in each group because of missing data.

2 Arithmetic mean (SEM), adjusted for BMI; education and dwelling are additionally adjusted for age and gender.

Table 2. Mean FFI scores by diet-related characteristics of the subjects $(n=306)$

\begin{tabular}{lrlc}
\hline Characteristics & $\mathrm{n}^{1}$ & $\mathrm{FFI}^{2}$ & $\mathrm{p}$ value \\
\hline Any dietary supplements & & & \\
$\quad$ No & 144 & $34.1(0.5)$ & \\
$\quad$ Yes & 136 & $35.4(0.5)$ & 0.04 \\
Good appetite & 32 & $33.5(1.0)$ & \\
$\quad$ No & 265 & $34.7(0.3)$ & 0.24 \\
$\quad$ Yes & & & \\
Some physical activity & 43 & $32.5(0.8)$ & \\
$\quad$ No & 254 & $34.9(0.3)$ & $<0.01$ \\
$\quad$ Yes & &
\end{tabular}

${ }^{1}$ Values are not always equal to total number of subjects in each group because of missing data.

2 Arithmetic mean (SEM), adjusted for age, gender and BMI.

\section{Methods}

Data from a qualitative 28 -item food-frequency questionnaire was used to calculate the FFI in a sample of 444 non-frail older adults, 382 women and 62 men, aged 55 years and older living in Vienna, Austria. The sample was drawn in September and Octo- ber 2001 from consecutive attendance to information events on healthy aging which took place in 10 nursing homes of the municipal of Vienna. Both free-living older adults and nursing home residents were invited. At the information events all eligible participants were provided with detailed information about the study and questionnaires including the 28-item FFQ were administered. Participants who were $<55$ years of age, who had mental disabilities or comprehension problems and who needed constant care were not eligible for the study. All participants, 55-98 years old, gave their written informed consent. The study was approved by the local ethics committee and conducted in accordance with the committee's rules.

All statistical analyses were performed with Stata statistical software, version SE 9.1. Multiple linear regression models were used to examine associations of diet quality (as measured with the FFI) with the selected factors.

To control for possible confounding, all models were a priori adjusted for age, gender, and body mass index (BMI). For the different categories of the subgroups the corresponding arithmetic mean and SEM of the FFI was calculated. All analyses excluded respondents with missing values. All regression models were tested for model assumptions and fit. No significant interaction was found for the subgroup-specific regression coefficients.

For the 433 subjects who reported their body weight and height, the body mass index (BMI) was calculated (weight in kilograms divided by the square of height in meters).

\section{Results}

The mean age of the subjects was 75 years (SD 11) (range: 55-98 years) and the mean BMI was 26.6 (SD 4.1) (range: 17.9-48.3). BMI decreased with increasing age $(-0.6$ for each 10 -year interval, $\mathrm{p}<0.001)$; no gender differences were observed in mean BMI.

In linear multiple regression models, diet quality was associated with several selected factors. A higher FFI, indicating a favorable diet quality, was obtained for women as compared to men, younger and better educated subjects ( $\mathrm{p}$ for all $<0.01$ ). No statistically significant associations were found between mean FFI scores and type of dwelling (living in a private home vs. in a nursing home) (table 1).

Dietary supplement users $(\mathrm{p}=0.04)$ and subjects being physically active $(\mathrm{p}<0.01)$ showed significantly higher FFI scores (table 2).

Eating difficulties $(\mathrm{p}=0.04)$ and gastrointestinal problems $(p=0.03)$ were found to be risk factors for poor diet quality as measured with the FFI, whereas the number of prescribed daily medications was not associated with diet quality ( $\mathrm{p}$ for trend $=0.77$ ) (table 3$)$. 
Table 3. Mean FFI scores by health characteristics of the subjects $(\mathrm{n}=306)$

\begin{tabular}{|c|c|c|c|}
\hline Characteristics & $\mathrm{n}^{1}$ & $\mathrm{FFI}^{2}$ & $\mathrm{p}$ value \\
\hline \multicolumn{4}{|c|}{ Eating difficulties } \\
\hline No & 248 & $34.9(0.4)$ & \\
\hline Yes & 50 & $33.1(0.8)$ & 0.04 \\
\hline \multicolumn{4}{|c|}{ Gastrointestinal problems } \\
\hline No & 205 & $35.0(0.4)$ & \\
\hline Yes & 69 & $33.3(0.7)$ & 0.03 \\
\hline \multicolumn{4}{|c|}{ Number of daily prescribed medications } \\
\hline None & 34 & $35.7(1.0)$ & \\
\hline 1 & 57 & $34.5(0.7)$ & 0.34 \\
\hline $2-3$ & 95 & $34.1(0.6)$ & 0.17 \\
\hline $4-6$ & 71 & $34.6(0.7)$ & 0.36 \\
\hline 6 or more & 41 & $35.3(0.9)$ & 0.77 \\
\hline
\end{tabular}

${ }^{1}$ Values are not always equal to total number of subjects in each group because of missing data.

2 Arithmetic mean (SEM), adjusted for age, gender and BMI.

\section{Discussion}

In the present analysis risk factors associated with poor diet quality in non-frail older adults have been identified using the FFI. Several observations confirmed findings as described in the literature.

Davenport et al. [4] found significant associations between their diet quality index and several population subgroups as defined by gender, age, education, exercise, body mass index and other factors. Similarly, in the study of Hann et al. [5] Healthy Eating Index (HEI) scores were significantly influenced by age, education and vitamin supplement use. Older age groups and less-educated women had lower HEI scores, indicating less healthy diets, whereas user of vitamin supplements had healthier diets. The latter being referred to as 'inverse supplement hypothesis'; i.e. individuals adhering to healthier diets are also more likely to consume dietary supplements as compared to individuals with less-favorable eating habits [6].

It is often mentioned that living arrangement of older adults, particularly living alone or frequently eating alone, may affect dietary intake. However, no such associations were found in the present subjects; no differences in diet quality were found between subjects living alone as compared to subjects living with a partner or with other family members.

Diet Quality in Non-Frail Older Adults
In older populations, higher rates of health problems may have a negative impact on diet through various pathways. For example, previous studies described a relationship between loss of appetite and impaired dietary intake.

Payette et al. [7] showed that appetite in an elderly population was significantly related to dietary intake. Study subjects who reported poor appetites were estimated to consume less energy per day than those reporting good appetite.

Loss of appetite is often also related to medication, an observation also confirmed in the present study (Spearman's rho $=-0.20 ; \mathrm{p}<0.001)$.

However, the direct relationship between use of medication and dietary intake is less studied. We found only one recent study examining the relationship between these two factors, which, however, found significant associations in a multivariate model [8].

In the present analysis the number of daily prescribed medications was not associated with diet quality and neither with total energy intake (adjusting for age and gender).

Interestingly, reported gastrointestinal problems were predictive for a lower diet quality in the present study, but were not for total energy intake.

On the other hand, Shahar et al. [8] identified gastrointestinal problems as a risk factor for reduced energy intake (in older women).

The main strength of the present study is that the FFI was validated with use of biochemical indicators (biomarkers) of diet quality in the same study sample [unpubl. data].

Potential limitations of the study should also be pointed out. The population from which the sample was drawn was not representative of the general population over 55 years of age, since, as outlined in the methods part, the subjects taking part were more likely to be interested in healthy aging. The subjects were, thus, probably more health conscious, with healthier lifestyles, including healthier dieting. Consequently, the potential to discrimination between subjects in terms of diet quality could have been degraded to some degree.

The FFQ used for calculating the FFI asked frequencies of consumption during the previous 3 months. Recall problems of the past diet should also be mentioned as a potential limitation of the FFQ method in older adults.

In conclusion, differences in diet quality are evident in subgroups of non-frail older adults living in Vienna as defined by societal factors, such as education, lifestyle-

Ann Nutr Metab 2008;52(suppl 1):43-46 
related factors, such as being physically active, and health-related factors, such as oral health. The identified factors should be considered in nutrition monitoring and potential dietary intervention programs, respectively.

\section{Disclosure Statement}

This study was financially supported by the 'Kuratorium Wiener Pensionisten-Wohnhäuser' (Board of Trustees Nursing Homes of Vienna), 'Lebensmitteluntersuchungsanstalt der Stadt Wien' (Food Control Institute of the Municipality of Vienna), and 'Gesundheitswesen und Soziales der Stadt Wien' (Health and Social Services of the Municipality of Vienna).

\section{References}

1 Patterson RE, Haines PS, Popkin BM: Diet Quality Index: capturing a multidimensional behavior. J Am Diet Assoc 1994;94:5764.

2 Kennedy ET, Ohls J, Carlson S, Fleming K: The Healthy Eating Index: design and applications. J Am Diet Assoc 1995;95:11031108.

3 Kant AK, Schatzkin A, Graubard BI, Schairer C: A prospective study of diet quality and mortality in women. JAMA 2000;283:21092115 .
4 Davenport M, Roderick P, Elliott L, Victor C, Geissler C: Monitoring dietary change in populations and the need for specific food targets; lessons from the North West Thames Regional Health Survey. J Hum Nutr Diet 1995;8:119-126.

5 Hann CS, Rock CL, King I, Drewnowski A Validation of the Healthy Eating Index with use of plasma biomarkers in a clinical sample of women. Am J Clin Nutr 2001;74:479486.
6 Kirk SFL, Cade JE, Barrett JH, Conner M: Diet and lifestyle characteristics associated with dietary supplement use in women. Publ Hlth Nutr 1998;2:69-73.

7 Payette H, Gray-Donald K, Cyr R, Boutier V: Predictors of dietary intake in a functionally dependent elderly population in the community. Am J Publ Hlth 1995;85:677-683.

8 Shahar D, Shai I, Vardi H, Fraser D: Dietary intake and eating patterns of elderly people in Israel: who is at nutritional risk? Eur J Clin Nutr 2003;57:18-25. 